\title{
An Innovative Performance of Refuge using Stowage Main Servers in Cloud Computing Equipment
}

\author{
S. Ravichandran ${ }^{1}$ and J. Sathiamoorthy ${ }^{2}$ \\ ${ }^{1}$ Professor, Department of Computer Science, Annai Fathima College of Arts \& Science, Madurai, Tamil Nadu, India \\ ${ }^{2}$ Associate Professor, Department of Software Applications, Thiruthangal Nadar College, Chennai, Tamil Nadu, India \\ E-mail: drravichandran6@gmail.com, jsathyam74@gmail.com
}

\begin{abstract}
Distributed computing has been imagined as the cutting edge engineering of IT Enterprise. It moves the application programming and information bases to the incorporated enormous server farms, where the administration of the information and administrations may not be completely dependable. There are various security issues for distributed computing as it envelops numerous innovations including networks, information bases, working frameworks, virtualization, asset planning, exchange the board, load adjusting, simultaneousness control and memory the executives. Putting away information in an outsider's cloud framework causes genuine worry over information secrecy. Hence, security issues for a large number of these frameworks and advancements are material to distributed computing. We propose a key worker encryption conspire and incorporate it with a decentralized deletion code with the end goal that a safe conveyed stockpiling key framework is defined respectively. Keywords: Secure Storage, Decentralized Storage System, Storage Key Server Encryption, SDL, NAS and Secure Cloud
\end{abstract}

\section{INTRODUCTION}

A few patterns are opening up the period of Cloud Computing, which is an Internet-based turn of events and utilization of PC innovation. The always less expensive and all the more remarkable processors, along with the "product as a help" (SaaS) registering administrations from information and programming that live exclusively on distant server farms. Albeit imagined as a promising help stage for the Internet, this new information stockpiling worldview in "Cloud" achieves many testing configuration issues which have significant impact on the security and execution of the general framework. Perhaps the greatest worry with cloud information stockpiling is that of information respectability confirmation at untrusted workers.

A capacity worker disappointment relates to a deletion mistake of the code word image. However long the quantity of disappointment workers is under the resilience limit of the deletion code, the message can be recuperated from the code word images put away in the accessible stockpiling workers by the deciphering cycle [1]. This gives a trade-off between the capacity size and the resistance edge of disappointment workers. A decentralized deletion code is an eradication code that freely registers each code word image for a message. Consequently, the encoding cycle for a message can be part into an equal errands of creating code word images. A decentralized deletion code is appropriate for use in a disseminated stockpiling framework. After the message images are shipped off capacity workers, every capacity worker freely figures a code word image for the got message images and stores it [2]. This completes the encoding and putting away cycle. The recuperation cycle is the equivalent.

Putting away information in an outsider's cloud framework causes genuine worry on information privacy. To give solid privacy to messages away workers, a client can scramble messages by a cryptographic strategy prior to applying a deletion code technique to encode and store messages. At the point when he needs to utilize a message, he needs to recover the code word images from capacity workers, interpret them, and afterward unscramble them by utilizing cryptographic keys [2]. There are three issues in the above clear coordination of encryption and encoding. Right off the bat, the client needs to do most calculation and the correspondence traffic between the client and capacity workers is high. Besides, the client needs to deal with his cryptographic keys. On the off chance that the client's gadget of putting away the keys is lost or bargained, the security is broken. At last, other than information putting away and recovering, it is difficult for capacity workers to straightforwardly uphold different capacities. For instance, stockpiling workers can't straightforwardly advance a client's messages to another. The proprietor of messages needs to recover, disentangle, decode and afterward forward them to another client.

Another significant worry among past plans is that of supporting unique information activity for cloud information stockpiling applications. In Cloud Computing, the distantly put away electronic information may not exclusively be gotten to yet in addition refreshed by the customers, e.g., through square alteration, cancellation and inclusion, and so forth In spite of the fact that there are numerous troubles looked by scientists, it is all around accepted that supporting unique information activity can be of imperative significance to the down to earth use of capacity revaluating administrations. Taking into account the vital job of public audit ability and information elements for cloud information stockpiling, we propose a productive development for the consistent reconciliation of these two parts in the convention plan. 
The significant security challenge with mists is that the proprietor of the information might not have control of where the information is put. This is since, supposing that one needs to misuse the advantages of utilizing distributed computing, one should likewise use the asset allotment and planning gave by mists [7]. Accordingly, we need to shield the information amidst un trusted measures.

This commitment can be summed up as follows:

1. We build a safe distributed storage framework that bolsters the capacity of secure information sending by utilizing a limit key worker encryption plot. The encryption plot underpins decentralized eradication codes over scrambled messages and sending activities over encoded a lot messages [6]. Our framework is profoundly appropriated where capacity workers freely encode and forward messages and key workers autonomously perform incomplete decoding.

2. We spur the public evaluating arrangement of information stockpiling security in Cloud Computing, and propose a convention supporting for completely unique information activities, particularly to help block inclusion, which is missing in most existing plans.

3. We demonstrate the security of our proposed development and legitimize the presentation of our plan through solid usage and examinations with the cutting edge.

\section{RELATED WORK}

The quickly survey disseminated capacity frameworks, key worker encryption plans, and trustworthiness checking instruments. Appropriated stockpiling frameworks. At the early years, the Network-Attached Storage (NAS) [7] and the Network File System (NFS) [8] give additional capacity gadgets over the organization with the end goal that a client can get to the capacity gadgets by means of organization association. A short time later, numerous enhancements for adaptability, strength, proficiency, and security were proposed [1],[2],[9]. A decentralized design for capacity frameworks offers great versatility, in light of the fact that a capacity worker can join or leave without control of CA.

To guarantee the security and trustworthiness for cloud information stockpiling under the previously mentioned enemy model, we expect to plan proficient instruments for dynamic information check and activity and accomplish the accompanying objectives:

Storage Rightness: to guarantee clients that their information are without a doubt put away suitably and kept flawless all the time in the cloud.

Fast Confinement of Information Blunder: to viably find the breaking down worker when information defilement has been identified.

Dynamic Information Uphold: to keep up a similar degree of capacity accuracy confirmation regardless of whether clients alter, erase or annex their information records in the cloud.

Dependability: to improve information accessibility against Byzantine disappointments, vindictive information adjustment and worker conniving assaults, for example limiting the impact brought by information mistakes or worker disappointments.

Lightweight: to empower clients to perform capacity accuracy checks with least overhead. Documentation and Preliminaries.

1. F - The information record to be put away. We accept that $F$ can be signified as a framework of m equivalent measured information vectors, each comprising of 1 squares. Information blocks are for the most part all around spoke to as components in Galois Field GF(2p) for $\mathrm{p}=8$ or 16

2. A - The dispersal network utilized for Reed-Solomon coding

3. G - The encoded record grid, which incorporates a bunch of $\mathrm{n}=\mathrm{m}+\mathrm{k}$ vectors, each comprising of $\mathrm{l}$ squares

4. fkey $(\cdot)$ - pseudorandom work (PRF), which is characterized as $\mathrm{f}:\{0,1\}_{-}$? key $\rightarrow \mathrm{GF}(2 \mathrm{p}$

5. $\operatorname{key}(\bullet)-$ pseudorandom stage (PRP), which is characterized as _ : $\{0,1\} \log 2(1)$ ?key $\rightarrow\{0,1\} \log 2(1)$

6. ver - a rendition number bound with the list for singular squares, which records the occasions the square has been altered. At first we expect ver is 0 for all information blocks ij - the seed for PRF, which relies upon the document name, block list I, the worker position $\mathrm{j}$ just as the discretionary square form number ver.

\section{PROBLEM STATEMENT}

In cloud information stockpiling framework, clients store their information in the cloud and at this point don't have the information locally. Accordingly, the rightness and accessibility of the information documents being put away on the appropriated cloud workers should be ensured. One of the major questions is to successfully identify any unapproved information alteration and debasement, perhaps because of worker bargain and additionally irregular Byzantine disappointments. Furthermore, in the circulated situation when such irregularities are effectively recognized, to discover which worker the information blunder lies in is likewise of incredible criticalness, since it tends to be the initial step to quick recuperate the capacity mistakes. To address these issues, our primary plan for guaranteeing cloud information stockpiling is introduced in this part [4]. The initial segment of the part is dedicated to a survey of fundamental apparatuses from coding hypothesis that are required in our plan for record dispersion across cloud workers. At that point, the holomorphic token is presented. The symbolic calculation work we are thinking about has a place with a group of general hash work [9], picked to 
safeguard the holomorphic properties, which can be entirely incorporated with the check of deletion coded information [8] [10]. Thusly, it is likewise told the best way to determine a test reaction convention for checking the capacity accuracy just as recognizing making trouble workers. At last, the methodology for record recovery and blunder recuperation dependent on deletion amending code is illustrated. Let $F=(F 1, F 2, F m)$ and Fi $=(f 1 i, f 2 i, f l i) ~ T(I \in$ $\{1, m\})$.

Here $\mathrm{T}$ (shorthand for render) means that each $\mathrm{Fi}$ is spoken to as a segment vector, and l signifies information vector size in squares. Every one of these squares are components of GF (2p). The deliberate format with equality vectors is accomplished with the data dispersal grid $\mathrm{A}$, got from an $\mathrm{m} \times(\mathrm{m}+\mathrm{k})$ Vander monde lattice [26]: By duplicating $\mathrm{F}$ by $\mathrm{A}$, the client acquires the encoded document.

$\mathrm{G}=\mathrm{F} \cdot \mathrm{A}=(\mathrm{G}(1), \mathrm{G}(2), \ldots, \mathrm{G}(\mathrm{m}), \mathrm{G}(\mathrm{m}+1), \ldots, \mathrm{G}(\mathrm{n}))=(\mathrm{F} 1$, F2, . . , Fm, G(m+1), . . ,G(n)), where $G(j)=(g(j) 1, g(j) 2$, $\ldots, \mathrm{g}(\mathrm{j}) \mathrm{l}) \mathrm{T}(\mathrm{j} \in\{1, \ldots, \mathrm{n}\})$. As seen, the increase repeats the first information record vectors of $F$ and the leftover part $(G(m+1), G(n))$ are k equality vectors created dependent on F.

To accomplish affirmation of information stockpiling rightness and information mistake limitation all the while, our plan completely depends on the pre-registered confirmation tokens. The fundamental thought is as per the following: before record dissemination the client preregisters a specific number of short confirmation tokens on individual vector $G(j)(j \in\{1 \ldots n\})$, every symbolic covering an arbitrary subset of information blocks. Afterward, at the point when the client needs to ensure the capacity rightness for the information in the cloud, he challenges the cloud workers with a bunch of arbitrarily created block lists. After accepting test, each cloud worker processes a short "signature" over the predefined squares and returns them to the client. The estimations of these marks should coordinate the relating tokens pre-registered by the client. In the interim, as all workers work over a similar subset of the files, the mentioned reaction esteems for honesty check should likewise be a substantial code word dictated by mystery lattice P.

The assailant examines put away messages away workers, the mystery keys of non-target clients, and the shared keys put away in key workers. Note that capacity workers' store all the safe key encryption and confirmation of deletion coded in $\mathrm{t} \mathrm{h}$ e flow research furnishes cloud information security alongside limits the repetition [5]. The dispersed key convention gives the limitation of information mistake in parallel outcomes about the capacity state across the disseminated administration in archetypes. Broad security and execution examination shows that the proposed plot is profoundly effective and tough against Byzantine disappointment, noxious information alteration assault, and even worker Collusion assaults. In distributed storage framework, organizations store their information in the distantly found information worker. As needs be, rightness of the information is guaranteed. Despite the fact that occasionally unapproved individual may alter or erase the information which prompts worker bargain and additionally arbitrary Byzantine disappointments, because it tends to be the initial step for quick recuperation of the capacity mistakes. The distributed storage frameworks propose a powerful and adaptable circulated plot with unequivocal unique information uphold for record appropriation across cloud workers. By figuring holomorphic token utilizing all inclusive hash work [7] which can be totally coordinated with the check of deletion coded information. Just as it distinguishes getting out of hand workers. At last, the method for record recovery and blunder recuperation dependent on eradication rectifying code is sketched out. It accomplishes affirmation for information stockpiling rightness and information mistake confinement, utilizing pre-figured token. Prior to sharing record appropriation utilizing pre-registers a specific number of most brief confirmation token are produced that will guarantee security for a square of information in a document in distributed storage. At the point when the client needs to ensure the capacity accuracy for the information in the cloud, he challenges the cloud workers with a bunch of arbitrarily created block files. In the wake of getting affirmation of the client it again requests verification by which the client is affirmed to be the confirmed client. After getting confirmation, each cloud worker figures a short "signature" over the predefined squares and returns them to the client[6]. The estimations of these marks should coordinate the relating tokens pre-processed by the client.

The Security Development Lifecycle (SDL) is a product improvement security affirmation measure comprising of security rehearses gathered by seven stages Investigation, Analysis, Logical plan, Physical plan, Implementation, Maintenance [8]. The distributed computing security dependent on completely stockpiling key worker encryption, and coordinate it with a protected decentralized code to shape a safe dispersed stockpiling framework, giving after effects of counts scrambled information without knowing the crude information on which the figuring was done, regarding the information classification All logarithmic setting, the hardness suspicion, a deletion code over types, and our methodology is clarified beneath: Bilinear guide. Let $\mathrm{G} 1$ and $\mathrm{G} 2$ be cyclic multiplicative groups 2 with a prime request $\mathrm{p}$ and $\mathrm{g} \in \mathrm{G} 1$ be a generator. A guide $\sim \mathrm{e}: \mathrm{G} 1 \times \mathrm{G} 1 \rightarrow \mathrm{G} 2$ is a bilinear guide on the off chance that it is proficiently process able and has the properties of linearity and non-decadence: for any $\mathrm{x}, \mathrm{y} \in$ $\mathrm{Z} * \mathrm{p}$, $\mathrm{e}(\mathrm{gx}, \mathrm{gy})=$ e (g, g)xy and $\mathrm{e}(\mathrm{g}, \mathrm{g})$ isn't the character component in G2. Let Gen $(1 \lambda)$ be a calculation producing ( $g$, e, G1, G2, p), Where $\lambda$ is the length of $p$. Let $x \in R \quad X$ signifies that $\mathrm{x}$ is haphazardly browsed the set $\mathrm{X}$. Decisional bilinear Diffie-Hellman presumption. This supposition that will be that it is computationally infeasible to recognize the appropriations (g, gx, gy, gz, e(g, g)xyz) and (g, gx, gy, gz, e(g, g)r), where $x, y, z, r \in R$ Z* p. Officially, for any probabilistic polynomial time calculation $A$, the 
accompanying is insignificant (in $\lambda)$ : $\mid \operatorname{Pr}[\mathrm{A}(\mathrm{g}, \mathrm{gx}, \mathrm{gy}$, gz,Qb) = b : x, y, z, r $\in$ R Z*p; Q0 = $\mathrm{e}(\mathrm{g}, \mathrm{g}) \mathrm{xyz} ; \mathrm{Q} 1=\sim \mathrm{e}(\mathrm{g}$, g) $r ; b \in R\{0,1\}]-1 / 2 \mid$ Erasure coding over types. We consider that the message area is the cyclic multiplicative gathering G2 depicted previously. An encoder produces a generator grid $\mathrm{G}=[$ gi,j ] for $1 \leq \mathrm{I} \leq \mathrm{k}, 1 \leq \mathrm{j} \leq \mathrm{n}$ as follows. For each column, the encoder arbitrarily chooses a passage and haphazardly sets an incentive from $\mathrm{Z} * \mathrm{p}$ to the passage. The encoder rehashes this progression $\mathrm{v}$ occasions with substitution for each column [10].

A section of a line can be chosen on numerous occasions yet simply set to one worth. The estimations of the rest passages are set to 0 . Leave the message alone (m1, m2, mk) Gk2. The encoding expert cuss is to create When client A needs to store a message of $\mathrm{k}$ squares $\mathrm{m} 1, \mathrm{~m} 2, \ldots$. . $\mathrm{mk}$ with the identifier ID, he processes the personality token $\tau=$ hf(a3,ID) and plays out the encryption calculation $\operatorname{Enc}(\bullet)$ on $\tau$ and $\mathrm{k}$ squares to get $\mathrm{k}$ unique code messages $\mathrm{C} 1, \mathrm{C} 2, \ldots$, Ck. A unique code text is shown by a main piece $b=0$. Client A sends each code text $\mathrm{Ci}$ to $\mathrm{v}$ arbitrarily picked capacity workers. A capacity worker gets a bunch of unique code messages with a similar personality token $\tau$ from A.

\section{IMPLEMENTATION}

We investigate capacity and calculation complexities, rightness, and security of our distributed storage framework in this segment. Give the spot length of a component access the gathering $\mathrm{G} 1$ be $\mathrm{l} 1$ and G2be 12 . Let coefficients gi,j be haphazardly browsed $\{0,1\} 13$. Capacity cost. To store a message of $\mathrm{k}$ squares, a capacity worker SSj stores a code word image $(b, \alpha j, \tau, \gamma j)$ and the coefficient vector $(g 1, j, g 2$, $\mathrm{j}, \mathrm{gk}, \mathrm{j})$. They are complete of $(1+2 \mathrm{l} 1+\mathrm{l} 2-\mathrm{kl} 3)$ bits, where $\alpha \mathrm{j}, \tau \in \mathrm{G} 1$ and $\gamma \mathrm{j} \in \mathrm{G} 2$. The normal cost for a message bit put away in a capacity worker is $(1+2 \mathrm{l} 1+\mathrm{l} 2-\mathrm{kl} 3) / \mathrm{kl} 2$ bits, which is overwhelmed by $13 / 12$ for an adequately enormous $\mathrm{k}$ [11].

By and by, little coefficients, for example $13<<12$, lessen the capacity cost in every capacity worker. Calculation cost. We measure the calculation cost by the quantity of matching activities, secluded exponentiations in G1 and G2, particular augmentations in G1 and G2, and number juggling tasks over GF (p). These tasks are meant as Pairing, Exp1, Exp2, Mult1, Mult2, and Fp, individually.

The expense is summed up in Table I. Processing a Fp takes significantly less time than figuring a Mult1 or a Mult2. The hour of processing an Exp1 is $0.75 \log$ p times as much as the hour of figuring a Mult1 all things considered (by utilizing the square-and duplicate calculation). Additionally, the hour of registering an Exp2 is 0.25log p times as much as the hour of processing a Mult2, all things considered (Pairing: a matching calculation of e. - Exp1 and Exp2: a particular exponentiation calculation in G1 and G2, separately. - Mult1 and Mult2: a measured increase calculation in G1 and G2, separately. - Fp: a numbercrunching activity in GF (p). Rightness.
TABLE I THE COMPUTATION COST OF EACH ALGORITHM IN SECURE CLOUD STORAGE SYSTEM

\begin{tabular}{|c|c|}
\hline Operation & Computation cost \\
\hline Enc & kPairing+kExp1+k Mult2 \\
\hline Encode & $\begin{array}{l}\text { kExp1+kExp2 +,(k-p)Mult1 +(k } \\
\text {-p)Mult2 } \\
\text { p)Mult1 }+(\mathrm{k}-\mathrm{p}) \text { Mult2 }\end{array}$ \\
\hline Key Recover & $\mathrm{O}(\mathrm{t}) \mathrm{Fp}$ \\
\hline Key Server Enc & 1Pairing+1Mult2 \\
\hline Key Server Dec & $\mathrm{t}-\mathrm{p}) \operatorname{Exp} 1$ \\
\hline
\end{tabular}

There are two cases for rightness. A Correctly recovers his message and client B effectively recovers a message sent to him. However long at any rate $\mathrm{k}$ stockpiling workers are accessible, a client can recover information with a mindboggling likelihood.

Accordingly, our capacity framework endures $\mathrm{n}-\mathrm{k}$ worker disappointments. An effective recovery is an occasion that a client effectively recovers all $\mathrm{k}$ squares of a message regardless of whether the message is possessed by him or sent to him [9]. The arbitrariness comes from the irregular determination of capacity workers in the information stockpiling stage, the arbitrary coefficients picked by capacity workers. The likelihood of an effective recovery relies upon (n, k, p).

\section{CONCLUSION}

In this paper, we consider a distributed storage framework comprises of capacity workers and key workers. We coordinate a recently proposed capacity key worker encryption conspire and eradication codes over examples. The capacity key worker encryption underpins encoding, sending, and fractional decoding tasks in an appropriated way. To unscramble a message of $\mathrm{k}$ squares that are scrambled and encoded to $n$ code word images, each key worker just needs to halfway decode 1 code word images in our framework. By utilizing the capacity key worker encryption plot, we present a protected distributed storage framework that gives secure information stockpiling and secure information sending usefulness in a decentralized structure. Also, every capacity worker freely performs encoding and encryption and each key worker autonomously performs' fractional unscrambling. Our key worker goes about as access hubs for giving a front end layer, for example, a conventional document framework interface. Further examination on itemized participation is required.

\section{REFERENCES}

[1] A. G. Dimakis, V. Prabhakaran and K. Ramchandran, "Ubiquitous access to distributed data in large-scale sensor networks through decentralized erasure codes," in Proceedingsofthe4th International Symposium on Information Processing in Sensor Networks-IPSN, IEEE Computer Society, pp. 111-117, 2018. 
[2] R. Bhagwan, K. Tati, Y. C. Cheng, S. Savage and G. M. Voelker, "Total recall: System support for automated availability management," in Proceedings ofthe1st Symposium on Networked Systems Design and Implementation - NSDI, pp.337-350, USENIX, 2019.

[3] A. Adya, W. J. Bolosky, M. Castro,G Cermak, R. Chaiken, J. R. Douceur, J. Howell, J. R. Lorch, M. Theimer, and R. Wattenhofer, "Farsite: Federated, available, and reliable storage for an incompletely trusted environment," in Proceedings of the 5th Symposium on Operating System Design and implementationOSDI, pp. 1-14, 2017.

[4] Z. Wilcox-O'Hearn and B. Warner, "Tahoe: the least-authority files system,” in Proceedingsofthe4th ACM International Workshop on Storage Security and Survivability-Storage SS, pp. 21-26, ACM, 2017.

[5] H. Y. Lin and W. G. Tzeng, "A secure decentralized Erasurecode for Distributed network storage," IEEE Transactions on Parallel and Distributed Systems, Vol. 21, pp.1586-1594, 2017.

[6] D. R. Brownbridge, L. F. Marshall and B. Randell, "The New castle connection or unixes of the world unite!" Software Practice and Experience, Vol. 12, No. 12, pp. 1147-1162, 2017.

[7] R. Sandberg, D. Goldberg, S. Kleiman, D. Walsh, and B. Lyon, "Design and implementation of the sun network file system," 2017.
[8] M. Kallahalla, E. Riedel, R. Swaminathan, Q.Wang, and K. Fu,"Plutus: Scalable secure files haring on untrusted storage, "in Proceedings of the 2ndUSENIX Conference on File and Storage Technologies-FAST, pp. 29-42, USENIX, 2018.

[9] S. C. Rhea, P. R. Eaton, D.Geels, H. Weatherspoon, B. Y. Zhao, and J. Kubiatowicz, "Pond: The ocean store prototype," in Proceedings of the 2nd USENIX Conference on File and Storage Technologies-FAST, pp.1-14, USENIX, 2019.

[10] John W. Rittinghouse, James F. Ransome, "Cloud Computing Implementation, Management, Security, CRCPress2018by Taylor and Francis Group, LLC.

[11] P. Druschel and A. Rowstron, "PAST: A large-scale, persistent peer-toper storage utility," in preceeding of the 8th Workshop on Hot topics in Operating System-Hot OS VIII pp.75-80, USENIX, 2019.

[12] A. Haeberlen, A. Mislove and P. Druschel, "Glacier: Highly durable, decentralized storage despite massive Symposium on Networked System Design and Implementation-NSDI, pp. 143158, USENIX, 2018. 